\title{
Mortalidade por doenças cardiovasculares em três estados do Brasil de 1980 a 2002
}

\author{
Gláucia M. M. de Oliveira, ${ }^{1}$ Carlos H. Klein ${ }^{2}$ e Nelson A. de Souza e Silva ${ }^{1}$
}

Como citar Oliveira GMM, Klein CH, Souza e Silva NA. Mortalidade por doenças cardiovasculares em três estados do Brasil de 1980 a 2002. Rev Panam Salud Publica. 2006;19(2):85-93

RESUMO Objetivo. Avaliar e comparar a mortalidade de adultos por doenças do aparelho circulatório (DAC), especialmente doenças isquêmicas do coração (DIC) e doenças cerebrovasculares (DCBV), no período de 1980 a 2002 nos Estados do Rio de Janeiro, Rio Grande do Sul e São Paulo e em suas capitais, levando em consideração o impacto dos óbitos por causas mal definidas nas taxas de mortalidade.

Métodos. Foram estimadas as taxas de mortalidade (brutas e ajustadas por sexo e idade) por $D A C, D I C$ e DCBV em individuos com 20 ou mais anos. Essas taxas foram compensadas com parte dos óbitos por causas mal definidas, na mesma proporção dos óbitos por DAC, DIC ou $D C B V$ em relação a todos os óbitos, excluídos os mal definidos. Também foram estimadas as médias e diferenças anuais das taxas de mortalidade compensadas e ajustadas com modelos de regressão linear. A população padrão foi a do Estado do Rio de Janeiro em 2000.

Resultados. O declinio anual das taxas compensadas e ajustadas de mortalidade por DAC em 100000 habitantes variou de -13,1 a -8,7 no Estado do Rio de Janeiro e no Município de São Paulo, respectivamente. Nas DIC, o declínio anual foi maior no Município e no Estado do Rio de Janeiro $(-5,0$ e $-4,5$, respectivamente), e menor no Estado do Rio Grande do Sul e no Município de São Paulo (-2,8 e $-2,7$, respectivamente). Nas DCBV, a variação observada foi de $-6,5$ a $-2,9$ no Estado do Rio de Janeiro e em Porto Alegre, respectivamente.

Conclusão. Tendo em vista que o declínio nas taxas de mortalidade compensadas e ajustadas por DAC, DIC e DCBV ocorreu depois de 1980, é pouco provável que tenha resultado do controle dos fatores de risco e implementação da revascularização do miocárdio. Essa queda poderia estar relacionada ao desenvolvimento econômico acentuado que a precedeu, com conseqüente melhoria das condições de vida e redução da exposição a infecções no período perinatal e na infância.

Palavras-chave Causa da morte, estatísticas de mortalidade/tendências, doenças cardiovasculares, Brasil.

A partir do final da década de 1950, iniciou-se um declínio da mortalidade

\footnotetext{
1 Universidade Federal do Rio de Janeiro (UFRJ), Faculdade de Medicina, Departamento de Clínica Médica, Rio de Janeiro (RJ), Brasil. Enviar correspondência para Gláucia M. M. de Oliveira no seguinte endereço: João Lira 128/101, Leblon, CEP 22430-210, Rio de Janeiro, RJ, Brasil. E-mail: glaucia@ mls.com.br

2 Fundação Oswaldo Cruz, Escola Nacional de Saúde Pública, Rio de Janeiro (RJ), Brasil.
}

por doenças do aparelho circulatório (DAC) nos países industrializados, principalmente nos Estados Unidos, Canadá, Austrália e países do Oeste da Europa (1). Apesar dessa tendência, as DAC ainda são as principais causas de morte nos países desenvolvidos ou em desenvolvimento $(2,3)$, inclusive no Brasil $(4,5)$. Em 2002, as DAC foram a principal causa de morte no Brasil
(31\% do total), tendo sido responsáveis por mais do que o dobro das mortes resultantes da segunda causa, os cânceres. Dentre as DAC destacaram-se as doenças cerebrovasculares (DCBV) e as doenças isquêmicas do coração (DIC), que em 2002 compuseram mais de $60 \%$ dos óbitos por DAC (6).

No Brasil, a tendência de queda nas taxas de mortalidade por DAC foi 
constatada no Estado e Município de São Paulo $(7,8)$ e nos municípios de Salvador (9), Goiânia (10) e Porto Alegre (11). Atribuiu-se essa redução ao melhor controle dos fatores de risco cardiovascular, especialmente da hipertensão arterial sistêmica, às novas técnicas diagnósticas, aos avanços no campo terapêutico e à melhoria das condições socioeconômicas (12-14).

Mansur et al. observaram quedas na mortalidade por DAC, DIC e DCBV na maioria das capitais de 11 estados brasileiros no período de 1979 a 1996 (4). O Rio de Janeiro e Porto Alegre apresentaram uma redução no risco de morte por DIC e DCBV. São Paulo apresentou um discreto aumento nas DIC na faixa etária de 30 a 39 anos em ambos os sexos e nas mulheres de 40 a 59 anos. Por outro lado, esses mesmos autores mencionam altas taxas de mortalidade por causas mal definidas nas regiões Nordeste, Norte e CentroOeste, que poderiam influenciar as tendências observadas para as taxas de mortalidade de causas definidas, como as DAC. Além disso, não consideram a mortalidade crescente por causas mal definidas no Rio de Janeiro a partir de 1990 nem o seu impacto sobre outros dados de mortalidade.

O objetivo do presente estudo foi avaliar e comparar a mortalidade de adultos por DAC, DIC e DCBV no período de 1980 a 2002 nos Estados do Rio de Janeiro, Rio Grande do Sul e São Paulo e em suas capitais, levando em consideração o impacto dos óbitos por causas mal definidas nos dados sobre mortalidade.

\section{MATERIAIS E MÉTODOS}

Foram estimadas as taxas de mortalidade por DAC, DIC, DCBV e causas mal definidas entre os adultos dos Estados do Rio de Janeiro, São Paulo e Rio Grande do Sul e de suas capitais no período de 1980 a 2002. Foram considerados adultos os indivíduos com 20 ou mais anos de idade.

Os dados referentes às populações foram obtidos do Instituto Brasileiro de Geografia e Estatística (IBGE) com base nos censos de 1980, 1991 e 2000 e na contagem populacional de 1996 (15). Foi utilizado o método de Lagrange para os cálculos das populações em $1^{\circ}$ de julho para os anos intercensitários de 1980 a 2002 (16).

Os dados relativos às freqüências anuais de mortes por DAC foram obtidos do sistema DATASUS (17). Para os óbitos de 1980 a 1995 foram utilizados os códigos da Nona Revisão da Classificação Internacional de Doenças (CID9) (18), ou seja: para DIC, de 410 a 414; DCBV, de 430 a 438; DAC, de 390 a 459; e causas mal definidas, de 780 a 799 . Para os óbitos a partir de 1996 foram utilizados os códigos da CID-10 (19), ou seja: DIC, de I20 a I25; DCBV, de I60 a I69; DAC, de I10 a I82.9; e causas mal definidas, de R00 a R99.

Os dados foram estratificados segundo sexo e grupos etários (20 a 29 anos; 30 a 39 anos; 40 a 49 anos; 50 a 59 anos; 60 a 69 anos; 70 a 79 anos e 80 anos ou mais). Calcularam-se as taxas de mortalidade brutas e ajustadas por sexo e idade. A população padrão, para o ajustamento pelo método direto, foi a dos adultos do Estado do Rio de Janeiro no ano de 2000, de acordo com os dados do censo (15).

Devido ao crescimento relevante da mortalidade por causas mal definidas no Estado do Rio de Janeiro, a partir de 1990, decidiu-se compensar os óbitos certificados pelas causas definidas DAC, DIC e DCBV com parte dos óbitos de causas mal definidas em cada grupo, de acordo com o sexo e a idade. Essas compensações foram feitas na mesma proporção dos óbitos por DAC, DIC ou DCBV em relação a todos os óbitos, excluídos os mal definidos. A suposição foi a de que a distribuição das causas de óbito entre os mal definidos é semelhante à distribuição dos óbitos por causas definidas. Esse procedimento foi utilizado em todos os estados e capitais. O procedimento pode ser resumido pela fórmula $X_{c}=X+M * X /(T-M)$, onde $X$ é o número de óbitos pela causa específica DAC, DIC ou DCBV, M é o número de óbitos por causas mal definidas, T é o número de óbitos por todas as causas, e $X_{c}$ é o número compensado de óbitos pela causa específica. Somente depois das compensações foram feitos os ajustes por padronização, resultando em taxas de mortalidade "compensadas e ajustadas".

Também foram estimadas as médias e diferenças anuais das taxas de mortalidade compensadas e ajustadas com modelos de regressão linear. Consideraram-se as taxas de mortalidade compensadas e ajustadas por DAC, DIC e DCBV como variáveis dependentes $Y$, e os anos-calendário do estudo como variáveis independentes $X$. Portanto, a média corresponde ao meio do período, de 1980 a 2002, e as diferenças anuais à inclinação da reta, ambos em escala de óbitos por 100000 habitantes. Os modelos ficaram assim representados: $\hat{Y}=a+b X$, onde $\hat{Y}$ é a taxa estimada compensada e ajustada, a é a taxa no início do período e b é a variação anual média (coeficiente de inclinação da reta), sendo X o ano (1980 corresponde a $X=0$ ). As proporções das variações totais explicadas pelos modelos são expressas em $\mathrm{r}^{2}$ (variação de 0 a 1). As porcentagens de variação anual foram calculadas a partir da razão b/média. Para o ajustamento de taxas e demais procedimentos quantitativos foi utilizado o programa Stata.

\section{RESULTADOS}

Os óbitos por causas mal definidas apresentaram uma distribuição desigual nos três estados e municípios. No Estado e no Município de São Paulo, essas taxas permaneceram constantes. O mesmo aconteceu em Porto Alegre, a não ser por um pico extravagante no ano de 1989. No Estado do Rio Grande do Sul, a mortalidade bruta por causas mal definidas caiu em mais de $50 \%$ da década de 1980 para a seguinte, porém observou-se ascensão a partir de 2000. Já no Estado do Rio de Janeiro, a mortalidade por causas mal definidas aumentou mais de 2 vezes, e no Município do Rio de Janeiro quadruplicou da primeira para a segunda década. Se na primeira década o Rio Grande do Sul apresentava as maiores taxas de mortalidade por causas mal definidas, a partir do início da década de 1990 o Estado e o Município do Rio de Janeiro passaram a apresentar as taxas mais 
elevadas, representando esses óbitos pouco mais de um décimo do total (figuras 1 e 2). No período de 1990 a 2002 as taxas do Município do Rio de Janeiro foram 10 vezes maiores do que as das outras duas capitais.

A tabela 1 apresenta as taxas brutas médias de mortalidade por DAC, DIC e DCBV no período de 1980 a 2002, as médias compensadas pelos óbitos mal definidos e as médias compensadas e ajustadas por sexo e idade, estimadas pelos modelos de regressão linear. Além disso, são apresentadas as diferenças anuais médias das taxas de mortalidade compensadas e ajustadas, assim como a relação entre essas diferenças e as médias do período. Os modelos lineares foram capazes de explicar a variância total de forma satisfatória $\left(r^{2}=0,83\right.$ a 0,96$)$.

Observamos que os declínios anuais estimados das taxas de mortalidade compensadas e ajustadas, tanto nas capitais quanto nos Estados do Rio de Janeiro, São Paulo e Rio Grande do Sul, variaram de $-13,1$ a $-8,7$ óbitos por 100000 habitantes para DAC no Estado do Rio de Janeiro e no Município de São Paulo, respectivamente. Para as DIC, o declínio foi mais acentuado e semelhante no Estado do Rio de Janeiro e em sua capital, assim como em Porto Alegre. O menor declínio ocorreu no Rio Grande do Sul e no Município de São Paulo: -2,8 e-2,7 óbitos por 100000 habitantes, respectivamente. Para as DCBV, os extremos da variação anual observada foram de $-6,5$ a $-2,9$ óbitos por 100000 habitantes, no Estado do Rio de Janeiro e em Porto Alegre, respectivamente (tabela 1). Em geral, houve declínio nas taxas de DAC, DIC e DCBV em todos os locais. Portanto, os percentuais de variação anual das taxas de mortalidade estimadas também apresentaram redução em todas as situações. As taxas de mortalidade médias do período de 1980 a 2002 (brutas, compensadas e compensadas e ajustadas) foram mais elevadas tanto no Estado do Rio de Janeiro, como em sua capital, quando comparadas aos demais locais (tabela 1).

As figuras de 3 a 6 apresentam a evolução temporal das taxas de mortalidade compensadas e ajustadas por

FIGURA 1. Taxas de mortalidade por causas mal definidas em adultos de 20 anos ou mais nos Estados do Rio de Janeiro, São Paulo e Rio Grande do Sul ${ }^{\mathrm{a}}$

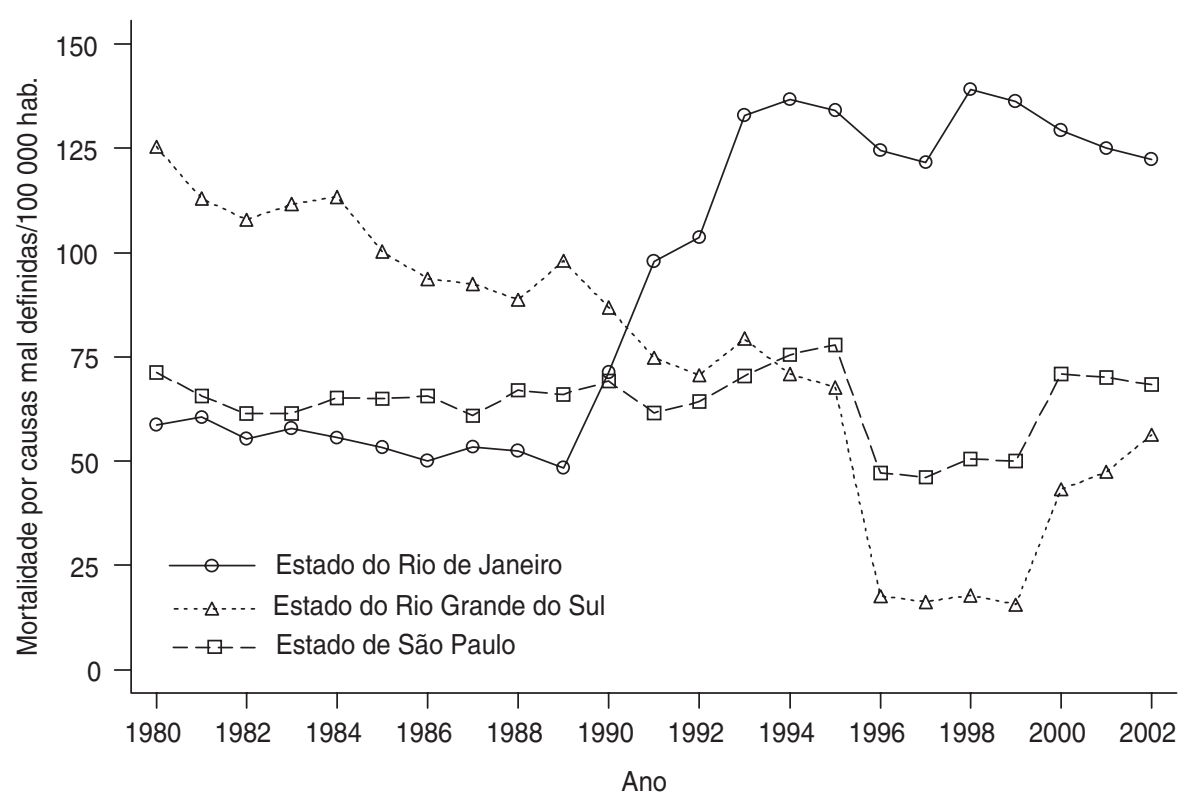

a Padrão: população do Estado do Rio de Janeiro em 2000. As taxas são ajustadas por sexo e idade.

FIGURA 2. Taxas de mortalidade por causas mal definidas em adultos de 20 anos ou mais nos municípios de Porto Alegre, Rio de Janeiro e São Pauloa

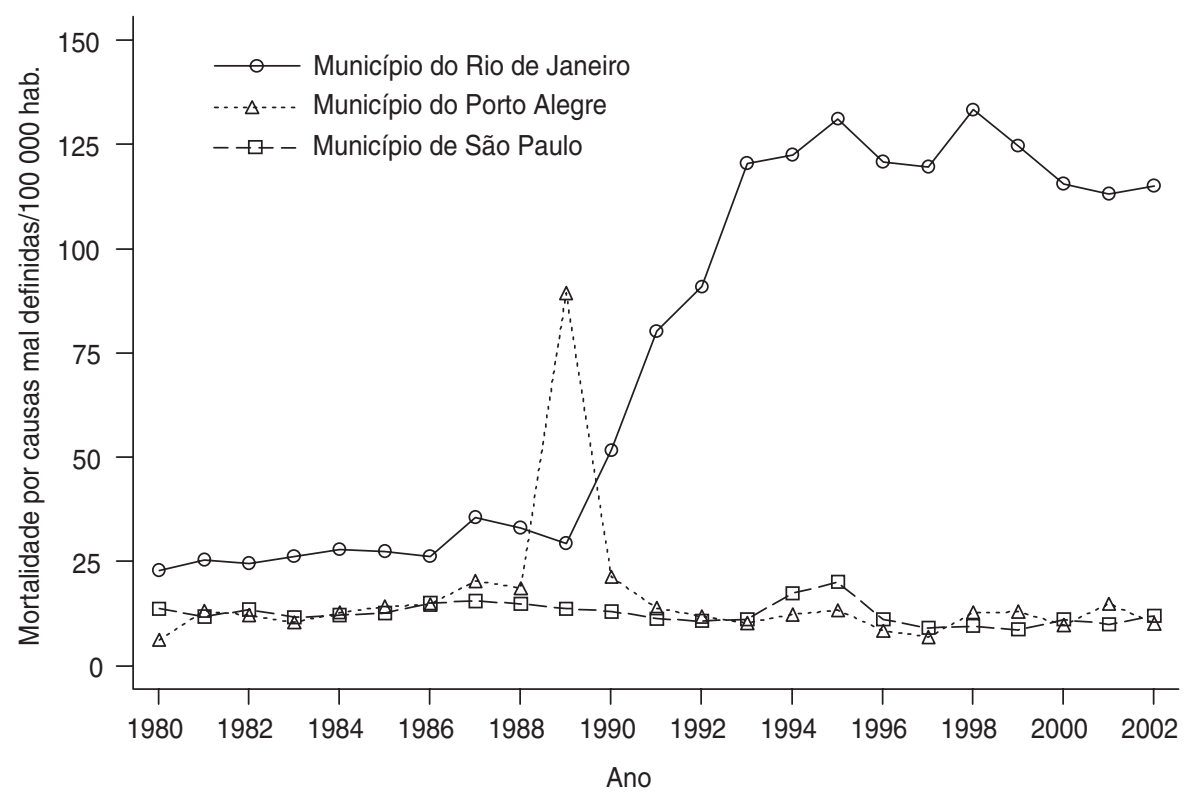

a Padrão: população do Estado do Rio de Janeiro em 2000. As taxas são ajustadas por sexo e idade.

DIC e DCBV nos estados e em suas capitais. Em relação à evolução da mortalidade por DIC, observaram-se números mais elevados no Estado do Rio de Janeiro em relação aos demais até 1991. A partir daí passou a haver equilíbrio (figura 3). Nas capitais não ocorreu um predomínio nítido, passando 
TABELA 1. Mortalidade cardiovascular em indivíduos com 20 anos ou mais nos Estados do Rio de Janeiro, Rio Grande do Sul e São Paulo e suas capitais, Brasil, 1980 a 2002

\begin{tabular}{|c|c|c|c|c|c|c|}
\hline \multirow[b]{2}{*}{ Causa $^{a}$} & \multicolumn{3}{|c|}{ Estados } & \multicolumn{3}{|c|}{ Capitais } \\
\hline & RJ & SP & RS & Rio & São Paulo & Porto Alegre \\
\hline \multicolumn{7}{|l|}{ Doenças isquêmicas } \\
\hline Média bruta & 142,0 & 118,2 & 131,1 & 165,1 & 133,8 & 158,0 \\
\hline Média compensada & 152,9 & 125,4 & 140,7 & 175,0 & 135,2 & 160,2 \\
\hline Média compensada e ajustada & 175,7 & 163,4 & 161,6 & 183,3 & 180,2 & 181,7 \\
\hline Diferença anual & $-4,5$ & $-3,4$ & $-2,8$ & $-5,0$ & $-2,7$ & $-4,4$ \\
\hline Diferença anual/média compensada e ajustada & $-2,6 \%$ & $-2,1 \%$ & $-1,7 \%$ & $-2,7 \%$ & $-1,5 \%$ & $-2,4 \%$ \\
\hline 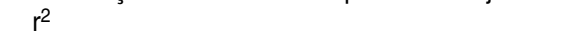 & 0,90 & 0,96 & 0,87 & 0,88 & 0,88 & 0,83 \\
\hline \multicolumn{7}{|l|}{ Doenças cerebrovasculares } \\
\hline Média bruta & 162,7 & 113,0 & 130,1 & 163,0 & 103,9 & 120,3 \\
\hline Média compensada & 175,4 & 119,8 & 140,4 & 172,7 & 105,0 & 122,0 \\
\hline Média compensada e ajustada & 206,0 & 159,4 & 166,2 & 182,8 & 141,2 & 138,0 \\
\hline Diferença anual & $-6,5$ & $-5,1$ & $-4,5$ & $-6,1$ & $-4,2$ & $-2,9$ \\
\hline Diferença anual/média compensada e ajustada & $-3,2 \%$ & $-3,2 \%$ & $-2,7 \%$ & $-3,4 \%$ & $-3,0 \%$ & $-2,1 \%$ \\
\hline$r^{2}$ & 0,96 & 0,96 & 0,94 & 0,96 & 0,93 & 0,85 \\
\hline \multicolumn{7}{|l|}{ Doenças do aparelho circulatório } \\
\hline Média bruta & 437,6 & 349,7 & 370,7 & 461,4 & 348,9 & 387,2 \\
\hline Média compensada & 472,1 & 370,8 & 399,1 & 489,8 & 352,6 & 392,7 \\
\hline Média compensada e ajustada & 547,3 & 487,4 & 466,7 & 513,5 & 469,5 & 443,6 \\
\hline Diferença anual & $-13,1$ & $-11,0$ & $-10,4$ & $-13,0$ & $-8,7$ & $-10,0$ \\
\hline Diferença anual/média compensada e ajustada & $-2,4 \%$ & $-2,3 \%$ & $-2,2 \%$ & $-2,5$ & $-1,9 \%$ & $-2,3 \%$ \\
\hline$r^{2}$ & 0,91 & 0,94 & 0,92 & 0,92 & 0,89 & 0,92 \\
\hline
\end{tabular}

FIGURA 3. Taxas de mortalidade por doenças isquêmicas do coração em adultos de 20 anos ou mais nos Estados do Rio de Janeiro, São Paulo e Rio Grande do Sula

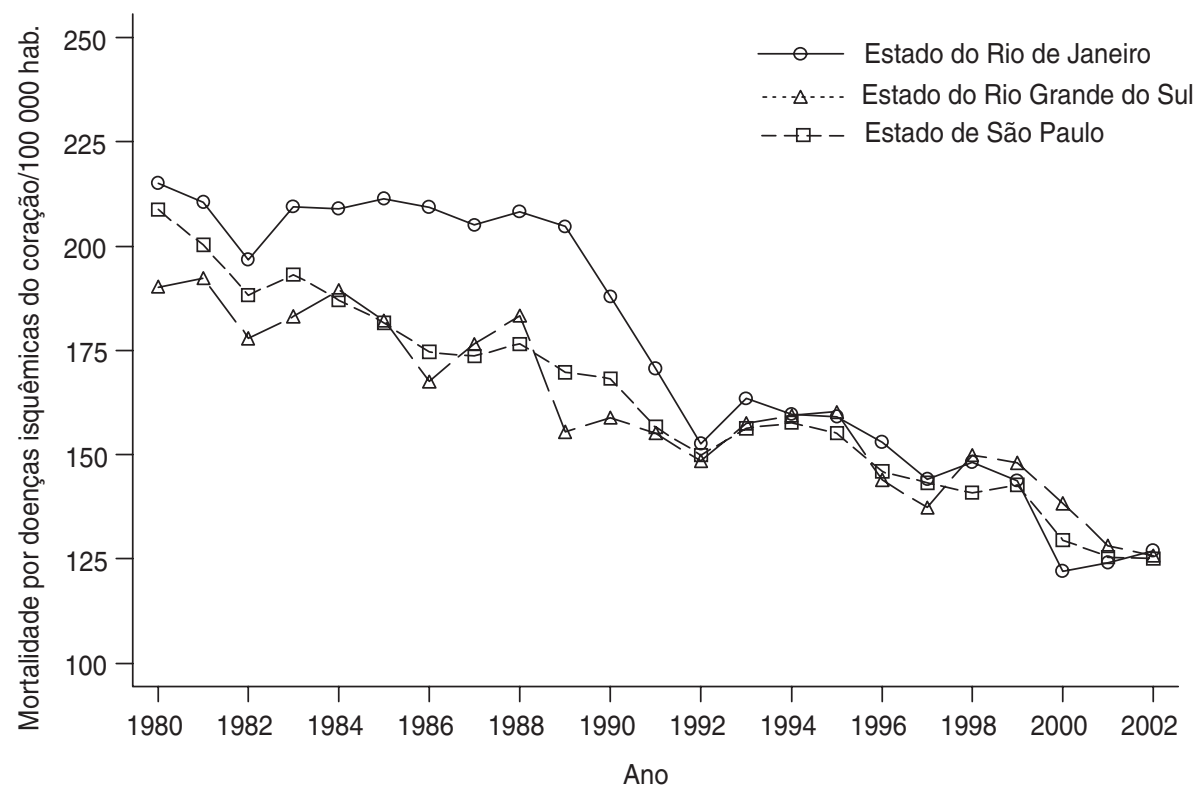

a Padrão: população do Estado do Rio de Janeiro em 2000. As taxas são ajustadas por sexo e idade e compensadas para minimizar o impacto do óbitos por causas mal definidas.

a capital do Rio de Janeiro a apresentar as taxas mais baixas no final do período, enquanto que São Paulo passou das taxas mais baixas nos primeiros anos para as mais elevadas no final do período (figura 4).
O Estado do Rio de Janeiro apresentou as maiores taxas compensadas e ajustadas de mortalidade por DCBV ao longo de todo o período estudado. Porém, a tendência de declínio dessas taxas nesse estado foi mais acentuada do que nos Estados de São Paulo e do Rio Grande do Sul (figura 5). Os Estados de São Paulo e Rio Grande do Sul apresentaram taxas de mortalidade por DCBV semelhantes ao longo dos anos estudados. Os mesmos padrões detectados nos estados, de redução das taxas por DCBV, se repetiram na comparação das capitais, porém as taxas do Município do Rio de Janeiro se aproximam mais das demais nos últimos anos (figura 6).

\section{DISCUSSÃO}

No Brasil foram publicados diversos estudos sobre a mortalidade por DAC com dados gerados a partir da década de 1970 (4, 5, 7-10, 12, 14). Laurenti (20), em 1981, sinalizou o aumento nas DIC, que representavam as principais causas básicas de morte $(16,7 \%)$ em 
adultos de 15 a 74 anos no Município de São Paulo. Em outro estudo, esse mesmo autor chamou a atenção para a preponderância das DCBV sobre as DIC em várias capitais brasileiras e para o fato de que se aproximavam das taxas de óbito por DCBV no Japão, mesmo no sexo masculino (21).

Chor et al. compararam as taxas de mortalidade no Brasil, Estados Unidos, Inglaterra e Cuba em 1988, e constataram que cerca de $50 \%$ dos óbitos masculinos por DIC ocorriam antes dos 65 anos de idade no Brasil, contra $25 \%$ nos outros países. Os autores ponderam que a população brasileira tem perfil mais jovem do que a dos demais países incluídos na comparação. $\mathrm{O}$ mesmo ocorreu em relação ao risco de morte por DCBV, que foi ainda maior no Brasil em comparação com os Estados Unidos, assemelhando-se ao de países do Leste Europeu (22).

De acordo com Lolio et al., que estudaram adultos maiores de 20 anos no Município de São Paulo, as taxas de mortalidade por DIC apresentaram tendência de aumento entre 1950 e 1976, seguida de declínio, especialmente no sexo feminino e nos mais jovens (23). De 1979 a 1989 observou-se um declínio desigual para a mortalidade por DIC e DAC em diversas capitais brasileiras (24). Nesse período, o Rio de Janeiro foi a única capital que apresentou tendência de aumento nas DAC e DIC.

Em nosso estudo, que se refere ao período de 1980 a 2002, o Estado e o Município do Rio de Janeiro apresentaram taxas de mortalidade por DAC e DCBV ajustadas e compensadas mais altas do que os Estados de São Paulo e Rio Grande do Sul e suas capitais. Por outro lado, a tendência de declínio nessas taxas foi mais acentuada no Estado e no Município do Rio de Janeiro. Na comparação, o Estado do Rio de Janeiro também apresentou as maiores taxas de mortalidade por DIC até o início da década 1990. Até esse ponto, as taxas de mortalidade por DIC se mantinham constantes no Estado e no $\mathrm{Mu}$ nicípio do Rio de Janeiro. Porém, a partir de então, as taxas de mortalidade por DIC no Estado do Rio de Janeiro foram se aproximando daquelas

FIGURA 4. Taxas de mortalidade por doenças isquêmicas do coração em adultos de 20 anos ou mais nos municípios do Rio de Janeiro, São Paulo e Porto Alegre ${ }^{a}$

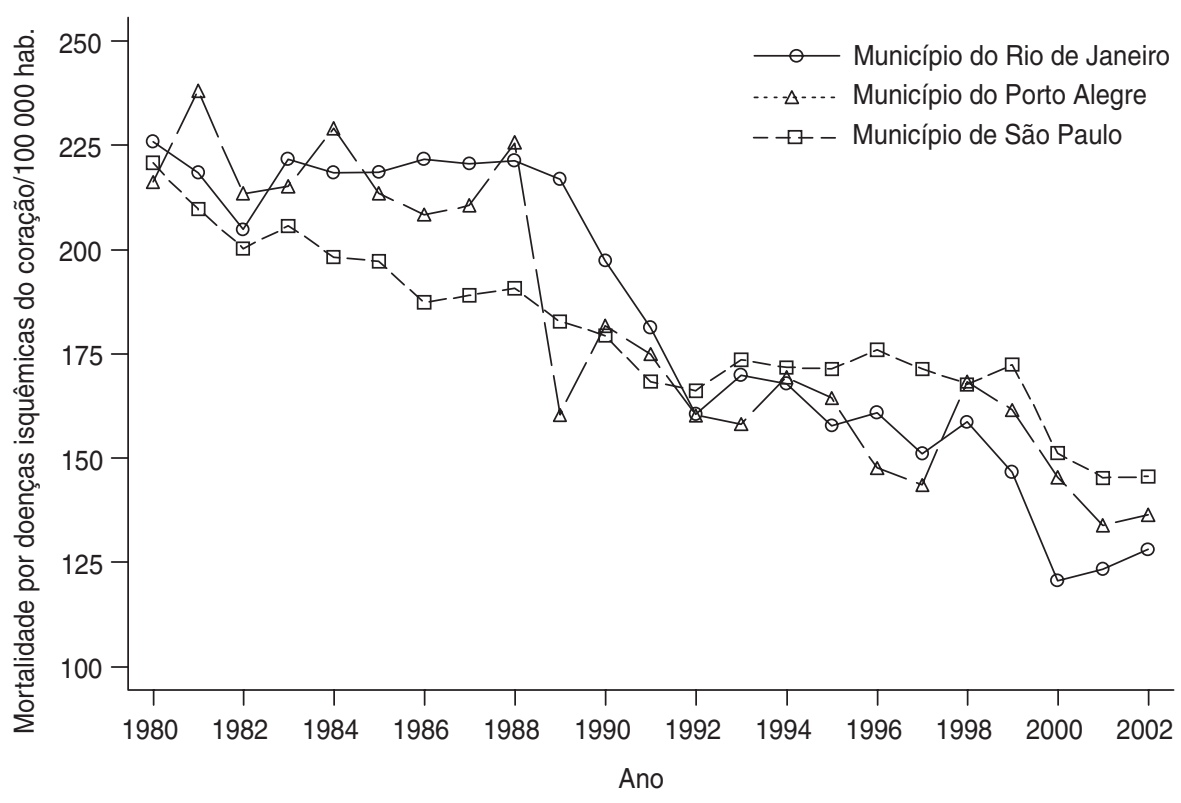

a Padrão: população do Estado do Rio de Janeiro em 2000. As taxas são ajustadas por sexo e idade e compensadas para minimizar o impacto do óbitos por causas mal definidas.

FIGURA 5. Taxas de mortalidade por doenças cerebrovasculares em adultos de 20 anos ou mais nos Estados do Rio de Janeiro, São Paulo e Rio Grande do Sula

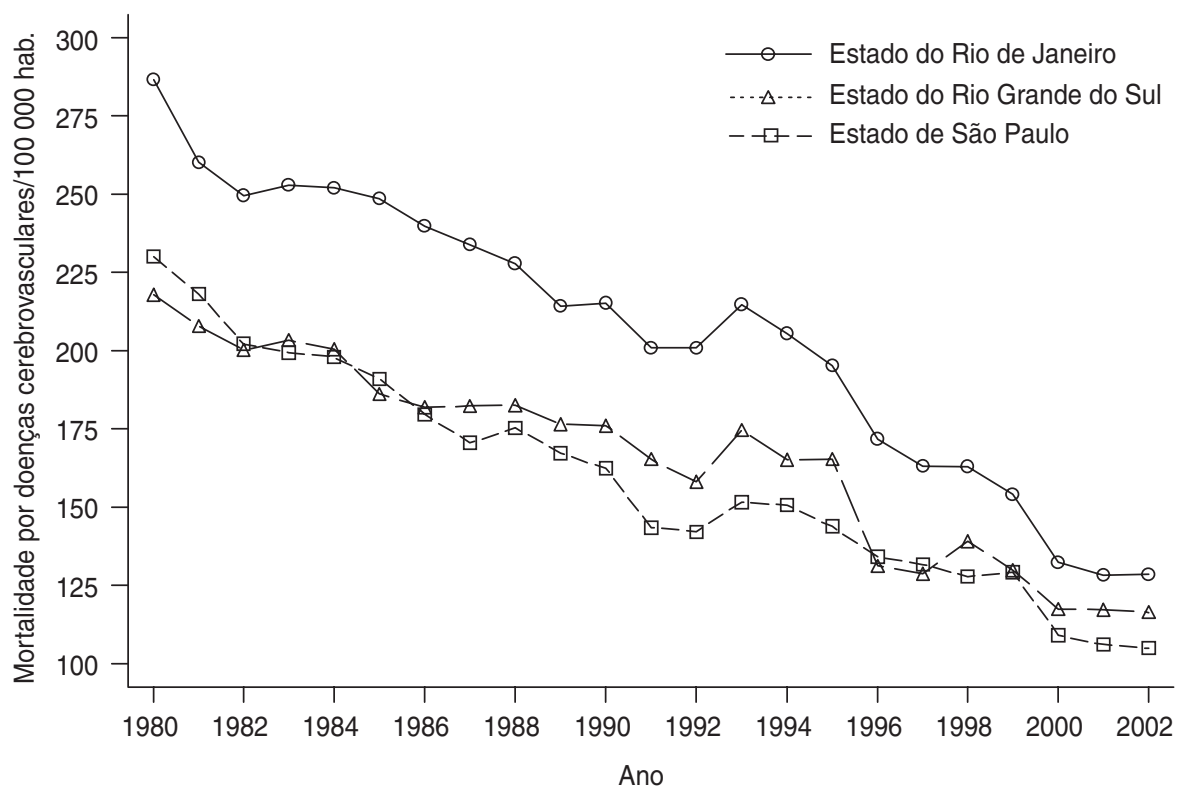

a Padrão: população do Estado do Rio de Janeiro em 2000. As taxas são ajustadas por sexo e idade e compensadas para minimizar o impacto do óbitos por causas mal definidas.

dos outros dois estados. Nas capitais não houve um predomínio claro quanto à mortalidade por DIC, ainda que, nos últimos anos, as taxas do $\mathrm{Mu}$ - nicípio do Rio de Janeiro tenham sido as mais baixas. Portanto, é possível supor que o aumento das taxas de mortalidade por DIC tenha ocorrido 
FIGURA 6. Taxas de mortalidade por doenças cerebrovasculares em adultos de 20 anos ou mais nos municípios do Rio de Janeiro, São Paulo e Porto Alegre ${ }^{a}$

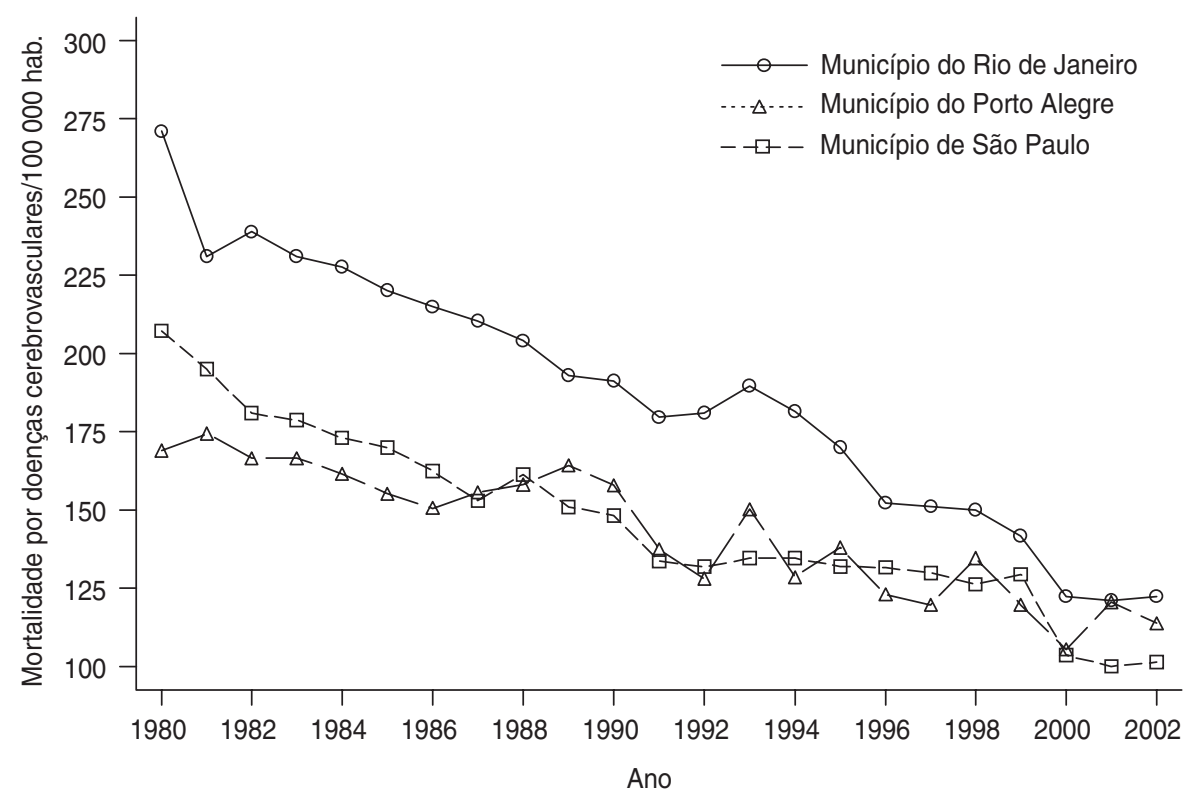

a Padrão: população do Estado do Rio de Janeiro em 2000. As taxas são ajustadas por sexo e idade e compensadas para minimizar o impacto do óbitos por causas mal definidas.

durante a década de 1970, com estabilização na década seguinte e queda a partir da década de 1990.

Mansur et al. observaram, no período de 1979 a 1996, uma tendência de queda na mortalidade por DAC, DIC e DCBV, em ambos os sexos, para o Brasil (4). Conforme esse estudo, o declínio das taxas de mortalidade por DIC ocorreu a partir de 1985, com queda progressiva até 1995, diferentemente dos Estados Unidos, onde a queda se iniciou em 1960 e continua desde então. Entretanto, a mortalidade por DCBV em homens e mulheres no Brasil foi sempre menor do que aquela registrada nos países do estudo MONICA $(4,13)$, discordando do que foi observado no Estado do Rio de Janeiro em nosso estudo.

Nos Estados Unidos, Canadá, Austrália e Nova Zelândia, a mortalidade por DAC aumentou a partir da década de 1940 até o início da década de 1960, quando se inicia a queda (1). O mesmo foi observado em países do Oeste da Europa, com o pico da mortalidade no final da década de 1960. No Brasil, assim como nos estados estudados por nós, a curva declinante é observada desde o início da década de 1980. O pico de mortalidade foi observado no final da década de $1970(7,8)$.

Antes de considerarmos as possíveis explicações para esses achados, chamamos a atenção para a qualidade das informações sobre mortalidade, a qual pode ser avaliada pela análise da mortalidade por causas mal definidas. Destacamos o aumento da mortalidade por essas causas no Estado e Município do Rio de Janeiro a partir de 1990, o que nos levou a calcular taxas de mortalidade compensadas, além de ajustadas por sexo e idade, para fazer as comparações entre os estados e os municípios. O aumento da mortalidade por causas mal definidas no Estado e no Município do Rio de Janeiro pode estar relacionado com uma portaria da Secretaria de Estado de Saúde, de 29 de janeiro de 1990, que determinou: "esgotadas todas as tentativas de se determinar a causa básica da morte e não havendo suspeita de óbito por causa violenta (acidente, homicídio ou suicídio), deverá ser declarada na parte 1 do atestado médico causa indeterminada" (25). Essa portaria pode ter influenciado os médicos no preenchi- mento de documentos de óbito sem diagnóstico definido no Rio de Janeiro.

E possível, também, que o procedimento que realizamos para compensar as perdas de óbitos por DAC e suas parcelas, DIC e DCBV, ainda tenha subestimado a verdadeira mortalidade por esses grupos de causas. Neste estudo consideramos que a proporção de óbitos por DAC entre os óbitos por causas mal definidas era a mesma do que a proporção de DAC em relação à mortalidade por todas as causas definidas, por sexo e idade. É possível que um número ainda maior de óbitos mal definidos devesse ter sido incorporado aos óbitos por DAC, DIC e DCBV como compensação, o que faria com que as tendências de queda por esses grupos de causas não fossem tão pronunciadas no Rio de Janeiro, especialmente quando comparadas às experimentadas por São Paulo, Rio Grande do Sul e suas capitais. São necessárias novas investigações que esclareçam as verdadeiras causas dos óbitos por causas mal definidas no passado e medidas que garantam o esclarecimento desses óbitos no futuro, como a instituição de centros de verificação de óbitos.

Um outro estudo (12) atribuiu a redução das taxas de mortalidade por $\mathrm{DCV}$ ao controle dos fatores de risco cardiovascular conhecidos (hipertensão arterial, tabagismo, obesidade, diabetes melito, dislipidemias e sedentarismo) e aos procedimentos de alta complexidade terapêutica (revascularização do miocárdio e uso de drogas). Entretanto, nos parece que, tanto no Rio de Janeiro como em outros locais, essas hipóteses não têm sustentação empírica para explicar a redução das taxas de mortalidade.

No Município do Rio de Janeiro, por exemplo, constatamos que a prevalência da hipertensão arterial sistêmica, segundo o estudo da Ilha do Governador, era de $38 \%$ na população adulta, e apenas $10 \%$ dos hipertensos tinham os seus níveis de pressão arterial controlados, enquanto que o tabagismo variava de cerca de $30 \%$ na população de alta renda até $50 \%$ nos homens de baixo nível socioeconômico (26). O bem estruturado Programa de Controle da Hipertensão Arterial do Muni- 
cípio do Rio de Janeiro (27), que já alcançou mais de 300 mil pacientes registrados em mais de 10 anos de atuação, controla apenas $19 \%$ da população-alvo de hipertensos. O diabetes, que afeta aproximadamente $8,7 \%$ das mulheres e $5,8 \%$ dos homens acima de 30 anos de idade, também apresenta baixas taxas de controle, principalmente na população de nível socioeconômico mais baixo, em que cerca de dois terços desconheciam a sua condição (28). A hipercolesterolemia atingia cerca de um terço da população adulta no interior do Estado do Rio de Janeiro (29). Sabe-se ainda que esses fatores de risco ocorrem mais freqüentemente na população de baixa renda e baixa escolaridade (30).

Na Europa, em estudo com pacientes pós-infarto do miocárdio, incluindo 15 países do Leste e do Oeste da Europa, o controle dos fatores de risco clássicos foi insatisfatório (31). Os programas de intervenção em comunidades obtiveram resultados conflitantes quanto à modificação dos fatores de risco clássicos para DAC e a conseqüente redução relevante das suas taxas de mortalidade (32). Além do mais, seria necessário que o controle desses fatores de risco se fizesse antes do reconhecimento da queda na mortalidade por DAC para que pudesse explicar os efeitos observados. Antes da década de 1960, nos países desenvolvidos, ou antes das décadas de 1970 e 1980, no Brasil, e mesmo a partir de então, esses fatores de risco possuíam um grau de controle ainda incipiente para justificar a redução observada na mortalidade cardiovascular. Apenas para o tabagismo conseguiu-se uma redução da prevalência em alguns países, ainda assim concentrada nos grupos de nível socioeconômico mais elevado. Para a obesidade (33) e para o sedentarismo, observa-se até um aumento de suas taxas de prevalência.

Portanto, com base nos dados disponíveis, não é possível explicar a progressiva queda nas taxas de mortalidade pelas doenças aterotrombóticas vasculares (coronarianas ou cerebrais) pelo maior controle dos fatores de risco clássicos, nem nos países desenvolvidos nem naqueles em desenvol- vimento. Os procedimentos de alta tecnologia (cirurgia de revascularização do miocárdio ou angioplastia) tampouco justificam as reduções de mortalidade observadas, uma vez que foram introduzidos depois de 1970. Nos países em desenvolvimento, essas técnicas não são acessíveis à grande maioria da população; além disso, seus resultados na prática clínica não parecem tão efetivos. No Estado do Rio de Janeiro, a letalidade hospitalar por revascularização do miocárdio foi, em média, 7,5\% entre 1999 e 2003 (34). Nos Estados Unidos, o declínio das taxas de mortalidade iniciou-se antes da introdução da cirurgia de revascularização do miocárdio, em 1967. Dessa forma, é improvável que esse procedimento tenha influenciado a queda na mortalidade cardiovascular. Além disso, em 2000 e 2001 (35), a letalidade associada à revascularização variou de $2,4 \%$ (em hospitais com mais de 450 cirurgias por ano) a 3,5\% (em hospitais com menos de 150 cirurgias por ano). Uma década antes, entre 1991 e 1993 (36), a letalidade pósoperatória imediata por essa cirurgia foi maior ainda, variando de 3,6\% (hospitais com mais de 100 cirurgias por ano) a 5\% (hospitais com menos de 100 cirurgias por ano). Os procedimentos de alta complexidade parecem pouco contribuir para a redução da mortalidade cardiovascular, tendo em vista que o tratamento clínico otimizado da cardiopatia isquêmica estável, com ou sem infarto do miocárdio prévio, resultou em mortalidade anual de apenas 0,8\% (37)_percentual esse de 3,0 a 6,3 vezes menor do que a letalidade imediata (e não anual) do procedimento invasivo. Também o estudo MONICA concluiu que o controle de fatores de risco clássicos explica apenas uma pequena proporção da variabilidade da mortalidade devida às doenças cardiovasculares (38).

Outros fatores, com sincronia temporal compatível com as tendências observadas na mortalidade cardiovascular nos diversos países, têm sido apontados pela literatura. Estudos demonstraram que o processo inflamatório tem papel primordial na etiopatogenia da aterosclerose. Os fatores de risco pró-inflamatórios, como as lipoproteínas de baixa densidade oxidadas, as citocinas próinflamatórias (interleucina 1 , fator de necrose tumoral $\alpha$ ), moléculas de adesão (seletinas, moléculas de adesão intercelular 1), estimuladores inflamatórios com efeitos hepáticos (como a interleucina 6) ou produtos da estimulação hepática (amilóide A sérico, proteína $\mathrm{C}$ reativa), entre outros, podem ser considerados marcadores da aterogênese ou preditores das complicações da doença aterosclerótica (39, 40). A redução da mortalidade por DAC também foi atribuída ao controle das infecções respiratórias por pneumonia e pelo vírus da influenza, principalmente nos meses de inverno, nos Estados Unidos, a partir de 1968 (41). Outra publicação recente aponta níveis menores de mortalidade por DAC nos primeiros 6 meses após uma única dose de vacina contra o vírus da influenza em pacientes portadores de infarto agudo do miocárdio (IAM) ou sintomáticos por angina que foram submetidos a procedimentos angiográficos (42). Espínola-Klein et al. demonstraram que níveis elevados de anticorpos para diversos agentes infecciosos, entre os quais a Chlamydia pneumoniae, os vírus de Epstein-Barr e herpes simples tipo 2, estavam associados à progressão da aterosclerose nas carótidas, mesmo após ajuste para idade, sexo e outros fatores de risco cardiovascular (43). Além disso, também o baixo peso ao nascer foi relacionado com o aumento da mortalidade cardiovascular e precoce na idade adulta $(44,45)$.

Dessa forma, podemos supor que a redução observada nas taxas de mortalidade cardiovascular poderia estar mais relacionada com a menor exposição a agentes infecciosos, principalmente nos primeiros anos de vida, devido às melhorias das condições de vida das populações, do que com o controle dos fatores de risco clássicos ou o avanço tecnológico introduzido na prática clínica. $\mathrm{O}$ crescimento econômico ocorrido no Brasil entre 1930 e 1980 (46), apesar de acompanhado por concentração de renda, possibilitou a disseminação de serviços de abastecimento de água e esgoto, com a conseqüente redução das doenças in- 
fecciosas e dos processos inflamatórios delas decorrentes (47).

A exposição a agentes infecciosos e outras condições insalubres nos primeiros anos de vida pode tornar os indivíduos mais suscetíveis ao desenvolvimento da aterotrombose vascular, e a elevada prevalência dos fatores de risco cardiovasculares clássicos, especialmente nas populações mais pobres (48-50), resulta em maior mortalidade por DAC, inclusive nos mais jovens (51). Por outro lado, a queda da mortalidade infantil precedeu por décadas a queda da mortalidade por DCV. A redução da mortalidade infantil foi atribuída ao controle da exposição às doenças infecciosas no período perinatal e nos primeiros anos de vida, com o declínio das mortes por pneumonia e diarréia infecciosa, aliado ao uso de vacinas. É possível também que a redução da exposição às doenças infecciosas nas fases iniciais da vida esteja relacionada com o declínio observado na mortalidade cardiovascular dos adultos. Em países desenvolvidos, esse declínio iniciou-se 15 anos após o término da segunda guerra mundial e pouco mais de 40 anos após a pandemia de influenza de 1918 (1). No Brasil, o declínio da mortalidade cardiovascular também começou pouco mais de 40 anos após o início do período de crescimento econômico (46).

As condições de moradia, a educação, o acesso à alimentação com base em frutas, legumes e verduras, o lazer, a inclusão social, os cuidados básicos de saúde, todos são aspectos ligados ao desenvolvimento econômico e social e certamente são fatores influentes na deter- minação das doenças infecciosas e também das doenças cardiovasculares (52). Lembramos que os índios Yanomami, que tivemos a oportunidade de estudar no Brasil (53), não apresentavam, na ocasião do estudo, hipertensão arterial ou diabetes melito, não eram obesos nem sedentários e seu colesterol total médio era menor que $140 \mathrm{mg} / \mathrm{dL}$. Para alcançar esses "resultados brilhantes" no controle dos fatores de risco cardiovascular não usavam nenhuma medicação. Portanto, a redução da mortalidade por doenças cardiovasculares deve estar mais relacionada à melhoria da qualidade de vida das populações desde o nascimento do que ao uso de drogas ou outros procedimentos de alta tecnologia, que devem ficar restritos aos casos de mais alto risco (acima de 20\%) onde sua efetividade é melhor.

\section{REFERÊNCIAS}

1. Le Fanu J. The case of the missing datacounselors and acolytes. BMJ. 2002;325(7378): 1490-3.

2. American Heart Association. Heart disease and stroke statistics. 2005 Update. Dallas, Texas: American Heart Association; 2005.

3. Strong K, Mathers C, Leeder S, Beaglehole R. Preventing chronic diseases: how many lives can we save? Lancet. 2005;366(9496): 1578-82.

4. Mansur AP, Favarato D, Souza MFM, Avakian SD, Aldrighi JM, Cesar LAM, et al. Tendência do risco de morte por doenças circulatórias no Brasil de 1979 a 1996. Arq Bras Cardiol. 2001;76(6):497-503.

5. Lotufo PA. Why Brazil does not have an outbreak of chronic diseases: lessons from cardiovascular diseases. Cienc Saude Coletiva. 2004;9(4):844-7.

6. Ministério da Saúde. Cadernos de Informação de Saúde. Mortalidade 2002. Disponível em: http://tabnet.datasus.gov.br/tabdata/ cadernos/BR/Brasil_GeralBR.xls. Acessado em dezembro de 2005 .

7. Lotufo PA, Lolio CA. Tendência da mortalidade por doença isquêmica do coração no Estado de São Paulo: 1970 a 1989. Arq Bras Cardiol. 1993;61(3):149-53.

8. Lolio CA, Souza JMP, Laurenti R. Decline in cardiovascular disease mortality in the city of São Paulo, Brazil, 1970 to 1983. Rev Saude Publica. 1986;20(6):454-64.

9. Passos LC, Lopes AA, Lessa I, Sanches A, Santos-Jesus R. Tendência da mortalidade por infarto do miocárdio (1981 a 1996) na cidade de Salvador, Brasil. Arq Bras Cardiol. 2000; 74(4):329-31

10. Moraes SA, Rezende MHV, Freitas ICM. Tendência da mortalidade por doença isquêmica do coração no município de Goiânia-
Brasil na série histórica entre 1980 e 1994 . Arq Bras Cardiol. 2000;74(6):493-7.

11. Estado do Rio Grande do Sul. Indicadores e dados básicos de saúde. Disponível em: http://www.saude.rs.gov.br/cronicos_ degenetarivos/cronicos_degenerativos.php. Acessado em janeiro de 2006.

12. Prata PR. The epidemiologic transition in Brazil. Cad Saude Publica. 1992;8(2):168-75.

13. Kuuslasmaa K, Tunstall-Pedoe H, Dobson A, Fortmann S, Sans S, Tolonen H, et al. Estimation of contribution of changes in classic risk factors to trends in coronary-event rates across the WHO MONICA Project populations. Lancet. 2000;355(9205):675-87.

14. Oliveira GMM, Klein $\mathrm{CH}$, Silva NAS. Análise crítica das mudanças das taxas de mortalidade por doenças do aparelho circulatório ocorridas entre 1980 e 2000, no Estado do Rio de Janeiro. Rev SOCERJ. 2003;16(2):95-100.

15. Instituto Brasileiro de Geografia e Estatística. Censo 2000. Disponível em: http://www. ibge.gov.br/censo/default.php. Acessado em dezembro de 2005

16. Cláudio DM, Martins JM. Cálculo numérico populacional. São Paulo: Atlas; 1989.

17. Brasil, Ministério da Saúde/Secretaria Executiva/Datasus. Informações de Saúde. Morbidade e informações epidemiológicas. Disponível em: http://w3.datasus.gov.br/ datasus $/$ datasus.php?area=359A1B378C5D0 E0F359G22H0I1Jd5L25M0N\&VInclude=.. / site/infsaude.php. Acessado em dezembro de 2005.

18. Organização Mundial de Saúde. Manual da Classificação Internacional de Doenças, Lesões e Causas de Óbitos. Nona revisão. São Paulo: Centro Colaborador da OMS para Classificação das Doenças em Português; 1978.
19. Organização Mundial de Saúde. Classificação Estatística Internacional de Doenças e Problemas Relacionados à Saúde. Décima revisão. São Paulo: EDUSP; 1995.

20. Laurenti R, Gotlieb SL, Souza JMP, Fonseca LAM, Jorge MHPM. Características da mortalidade por doença isquêmica do coração em adultos de 15 a 74 anos no município de São Paulo. Arq Bras Cardiol. 1981;36(2):85-9.

21. Laurenti R. Epidemiologia das doenças cardiovasculares no Brasil. Arq Bras Cardiol. 1982;38(4):243-8

22. Chor D, Fonseca MJ, Andrade CR. Doenças cardiovasculares. Comentários sobre a mortalidade precoce no Brasil. Arq Bras Cardiol. 1995;64(1);15-9.

23. Lolio CA, Laurenti R. Mortalidade por doença isquêmica do coração no município de São Paulo. Evolução de 1950 a 1981 e mudanças recentes na tendência. Arq Bras Cardiol. 1986; 46(3):153-6.

24. Lolio CA, Lotufo PA, Lira AC, Zanetta DM, Massad E. Tendência da mortalidade por doença isquêmica do coração nas capitais de regiões metropolitanas do Brasil, 1979-89. Arq Bras Cardiol. 1995;64(3):195-9.

25. Estado do Rio de Janeiro. Portaria ${ }^{\circ} 550$ de 23 de janeiro de 1990. Diário Oficial 1990. 29 de janeiro.

26. Klein $\mathrm{CH}$, Silva NAS, Nogueira AR, Campos LHS, Bloch KV. Hipertensão arterial na Ilha do Governador, Brasil. II. Prevalência. Cad Saude Publica. 1995;11(3):389-94.

27. Ramos ACMF, Seixas TC, Rocha CRM, Monteiro GT, Farias AMR. Avaliação transversal do controle da hipertensão arterial sistêmica em programa de larga escala. JBM. 2001;81(1): 63-70.

28. Franco L. Diabetes in Brazil: a review of recent survey data. Ethn Dis. 1992;2(2):158-65. 
29. Souza LJ, Chalita FEB, Reis AFF, Teixeira CL, Giocavate Neto C, Bastos DA, et al. Obesidade e fatores de risco cardiovascular em Campos, RJ. Arq Bras Cardiol. 2002;79(supl III):70.

30. Brandão AA, Magalhães EC, Pozzan R, et al. O nível socioeconômico e os fatores de risco cardiovasculares em jovens acompanhados por 10 anos. Estudo do Rio de Janeiro. Arq Bras Cardiol. 2002;79(supl. III):18.

31. Lifestyle and risk factor management and use of drug therapies in coronary patients from 15 countries. Principal results from EUROASPIRE II Euro Heart Survey Programme. Eur Heart J. 2001;22(7):554-72.

32. 33rd Bethesda Conference-Preventive cardiology: How can we do better? J Am Coll Cardiol. 2002;40(4):579-651.

33. Haslam DW, James WPT. Obesity. Lancet. 2005;366(9492):1197-1209.

34. Godoy PH, Klein CH, Souza e Silva NA, Oliveira GMM, Fonseca TMP. Letalidade na cirurgia de revascularização do miocárdio no Estado do Rio de Janeiro-SIH/SUS - no período de 1999-2003. Rev SOCERJ. 2005;18(1): 23-9.

35. Peterson ED, Coombs LP, DeLong ER, Haan CK, Ferguson TB. Procedural volume as a marker of quality for CABG surgery. JAMA. 2004;291(2):195-201.

36. Clark RE, and the Ad Hoc Committee of cardiac surgery credentialing of the society of thoracic surgeons: outcome as a function of annual coronary artery bypass graft volume. Ann Thorac Surg. 1996;61(1):21-6.

37. Jabbour S, Young-Xu Y, Graboys TB, Blatt CM, Goldberg RJ, Bedell SE, et al. Long-term outcomes of optimized medical management of outpatients with stable coronary artery disease. Am J Cardiol. 2004;93(3):294-9.
38. MONICA Monograph and Multimedia Sourcebook. Tunstall-Pedoe H, editor. Prepared by Tunstall-Pedoe H, Kuulasmaa K, Tolonen $\mathrm{H}$, Davidson M, Mendis S with 64 other contributors for The WHO MONICA Project. MONICA Monograph and Multimedia Sourcebook. Genebra: WHO; 2003. Disponível em http://www.ktl.fi/monica/public/monograph. $\mathrm{html}$. Acessado em dezembro de 2005.

39. Ridker PM, Rifai N, Rose L, Buring JE, Cook NR. Comparison of C-reactive protein and low-density lipoprotein cholesterol levels in the prediction of first cardiovascular events. N Engl J Med. 2002;347(20):1557-65.

40. AHA/CDC Scientific Statement. Markers of inflammation and cardiovascular disease application to clinical and public health practice. A statement for healthcare professionals from the centers for disease control and prevention and the American Heart Association. Circulation. 2003;107(3):499-511.

41. Cooper R, Stamler J, Dyer A, Garside D. The decline in mortality from coronary heart disease, U.S.A., 1968-1975. J Chronic Dis. 1978; 31(12):709-20

42. Gurfinkel E, Mautner B. Prevención secundaria de la cardiopatía isquémica con la vacuna antigripal. Nueva evidencia sobre el papel de la infección y los síndromes coronarios agudos. Rev Esp Cardiol. 2002;55(10):1009-12.

43. Espínola-Klein C, Rupprecht HJ, Blankenberg S, Bickel C, Kopp H, Victor A, et al. Impact of infectious burden on progression of carotid atherosclerosis. Stroke. 2002;33(11): 2581-6

44. Vaessen N, Janssen JA, Heutink P, Hofman A, Lamberts SW, Oostra BA, et al. Association between genetic variation in the gene for insulin-like growth factor-I and low birth weight. Lancet. 2002;359(9311):1036-7.

45. Barker DJ, Winter PD, Osmond C, Margetts B, Simmonds SJ. Weight in infancy and death from ischaemic heart disease. Lancet. 1989; 2(8663):577-80.

46. Lesbaupin I, Mineiro A. O desmonte da nação em dados. Petrópolis, Vozes, 2002.

47. Finch CE, Crimmins EM. Inflammatory exposure and historical changes in human lifespans. Science. 2004;305(5691):1736-9.

48. Bloom G. Equity in health in unequal societies: towards health equities during rapid social changes. IDS Working Paper 112; 2000.

49. World Development Report 2000/2001Attacking poverty. Oxford University Press / World Bank 2000.

50. United Nations Economic and Social Council. Report of the Secretary General: health and sustainable development; 2001.

51. Farrel R. Income inequality and cardiovascular disease in North-America: Shifting the paradigm. Harvard Health Policy Review. 2002;2(3). Disponível em http://www.hcs. harvard.edu. Acessado em dezembro de 2005.

52. Social Determinants of Health. The solid facts. Wilkinson R, Marmot M, editors. WHO 2003, 2nd ed.

53. Mancilha-Carvalho JJ, Silva NAS, Carvalho JV, Lima JAC. Pressão arterial em seis aldeias Yanomami. Arq Bras Cardiol. 1991;56(6): $477-82$.

Manuscrito recebido em 18 de junho de 2005. Aceito em versão revisada em 14 de novembro de 2005.
ABSTRACT

\section{Mortality from cardiovascular diseases in three Brazilian states from 1980 through 2002}

Objective. To evaluate and compare adult mortality from diseases of the circulatory system (CDs), especially ischemic heart disease (IHD) and cerebrovascular disease (CVD), from 1980 through 2002 in the Brazilian states of Rio de Janeiro, Rio Grande do Sul, and São Paulo and their capital cities (respectively Rio de Janeiro, Porto Alegre, and São Paulo), taking into account the impact of deaths due to ill-defined causes on mortality rates.

Method. We estimated mortality rates (crude and adjusted by age and sex) from CDs overall and from IHD and CVD among individuals aged 20 years or older. These rates were weighted with a portion of the deaths from ill-defined or unknown causes, in the same proportion as deaths from CDs, IHD, and CVD in relation to deaths overall, excluding deaths from ill-defined causes. Using linear regression models, we also estimated the mean values of and annual differences in the weighted adjusted mortality rates. The reference population was that of the state of Rio de Janeiro in 2000.

Results. The annual decline in the weighted adjusted mortality rates from CDs ranged from -13.1 per 100000 individuals in the state of Rio de Janeiro to -8.7 per 100000 in the city of São Paulo. For IHD, the annual declines were greatest in the city of Rio de Janeiro (-5.0 per 100000$)$ and the state of Rio de Janeiro (-4.5 per 100000$)$, and smallest in the state of Rio Grande do Sul (-2.8 per 100000$)$ and the city of São Paulo (-2.7 per 100 000). With CVD, the range that was found extended from -6.5 per 100000 in the state of Rio de Janeiro to -2.9 per 100000 in the city of Porto Alegre. Conclusion. The decreases in weighted adjusted mortality rates from CDs, IHD, and CVD occurred after 1980, so it is unlikely that the declines resulted from controlling risk factors or from the practice of myocardial revascularization. The decreases might be related to a period of strong economic development preceding the declines, which translated into improved living conditions and reduced exposure to infections in the perinatal period and childhood.

Key words Cause of death, mortality/trends, cardiovascular diseases, Brazil. 